\title{
SET-THEORETIC COMPLETE INTERSECTIONS ${ }^{1}$
}

\author{
T. T. $\mathrm{MOH}$
}

ABSTRACT. In this article we establish that:

(1) Every monomial curve in $\mathbf{P}_{h}^{\prime \prime}$ is a set-theoretic complete intersection, where $k$ is a field of characteristic $p$ (and thus generalize a result of $\mathrm{R}$. Hartshorne [3]).

(2) Let $k$ be an algebraically closed field of characteristic $p$ and $C$ a curve of $\mathbf{P}_{k}^{\prime \prime}$. If there is a linear projection $\tau: \mathbf{P}_{h}^{\prime \prime} \rightarrow \mathbf{P}_{h}^{2}$ with center of $\tau$ disjoint of $C, \tau(C)$ is birational to $C$ and $\tau(C)$ has only cusps as singularities, then $C$ is a set-theoretic complete intersection (and thus generalize a result of D. Ferrand [2]).

1. Let $\left(t^{d}, t^{a_{1}} u^{b_{1}}, \ldots, t^{a_{n} \cdot 1} u^{b_{n} \cdot 1}, u^{d}\right)$ with $n \geqslant 2$ be a parametrization of a curve $C$ in $\mathbf{P}_{h}^{\prime \prime}$, where $d=a_{1}+b_{1}=\cdots=a_{n-1}+b_{n-1}$ and $k$ is a field of characteristic $p$. The curve $C$ is said to be a monomial curve. Without losing generality we may assume that

$$
d>a_{1}>a_{2}>\cdots>a_{n-1}>0 .
$$

Let $\tau: k\left[x_{1}, \ldots, x_{n}\right] \rightarrow k[t]$ be the mapping defined by

$$
\tau\left(x_{1}\right)=t^{d}, \quad \tau\left(x_{2}\right)=t^{a_{1}}, \ldots, \tau\left(x_{n}\right)=t^{a_{n-1}}
$$

and $P=\operatorname{ker} \tau, Q=P \cap k\left[x_{1}, \ldots, x_{n-1}\right]$.

Given $f\left(x_{1}, \ldots, x_{n}\right) \in k\left[x_{1}, \ldots, x_{n}\right]$, let the highest homogeneous form of $f\left(x_{1}, \ldots, x_{n}\right)$ be denoted by $\partial f\left(x_{1}, \ldots, x_{n}\right)$. We shall establish

THEOREM 1. There are binomials $f_{1}, \ldots, f_{n-1} \in k\left[x_{1}, \ldots, x_{n}\right]$ such that

$$
\begin{gathered}
\sqrt{\left(f_{1}, \ldots, f_{n-1}\right)}=P, \\
\sqrt{\left(\partial f_{1}, \ldots, \partial f_{n-1}\right)}=\left(x_{2}, \ldots, x_{n}\right) .
\end{gathered}
$$

Proof. We shall use induction. The theorem is evident for $n=2$. We may assume that there are binomials $f_{1}, \ldots, f_{n-2} \in k\left[x_{1}, \ldots, x_{n-1}\right]$ such that

$$
\begin{gathered}
\sqrt{\left(f_{1}, \ldots, f_{n-2}\right)}=Q \\
\sqrt{\left(\partial f_{1}, \ldots, \partial f_{n-2}\right)}=\left(x_{2}, \ldots, x_{n-1}\right) .
\end{gathered}
$$

We shall construct $f_{n-1}$. Let

$$
g=\operatorname{GCD}\left(d, a_{1}, \ldots, a_{n-2}\right), \quad e=\operatorname{GCD}\left(a_{n-1}, g\right), \quad a_{n-1}=a_{n-1}^{*} e, \quad g=g^{*} e .
$$

Received by the editors December 16, 1983 and, in revised form. August 28, 1984.

198() Mathenatics Subject Classification. Primary 14M10.

'This work was partially supported by N.S.F. at Purdue University. 
Then the positive semigroup generated by $d, a_{1}, \ldots, a_{n-2}$ contains all large integers which are multiples of $g$. Hence there are integers $m, l_{1}, \ldots, l_{n-1}$ such that

$$
p^{m} g^{*} a_{n-1}=l_{1} d+\sum_{i=2}^{n-1} l_{i} a_{i-1} \text {. }
$$

In other words,

$$
f_{n-1}=\left(x_{n}^{g^{*}}\right)^{p^{m}}-x_{1}^{l_{1}} \prod_{i=2}^{n-1} x_{i}^{l_{i}} \in P .
$$

Clearly $p^{m} g^{*}>\sum_{i=1}^{n-1} l_{i}$. Hence we have

$$
\partial f_{n-1}=\left(x_{n}^{g^{*}}\right)^{p^{m}} \text { and } \sqrt{\left(\partial f_{1}, \partial f_{2}, \ldots, \partial f_{n-1}\right)}=\left(x_{2}, \ldots, x_{n}\right) \text {. }
$$

The conclusion (2) has been established. Let $h\left(x_{1}, \ldots, x_{n}\right)$ be any weighted homogeneous polynomial in $P$ with $x_{1}, \ldots, x_{n}$ of weight $d, \ldots, a_{n-1}$, respectively. Let

$$
h\left(x_{1}, \ldots, x_{n}\right)=\sum a_{i_{1} \ldots, i_{n}} x_{1}^{i_{1}} \cdots x_{n}^{i_{n}} .
$$

Then $i_{1} d+i_{2} a_{1}+\cdots+i_{n} a_{n-1}=$ const. It follows at once that

$$
i_{n} a_{n-1} \equiv i_{n}^{\prime} a_{n-1} \quad(\bmod g)
$$

for two indices $i_{n}, i_{n}^{\prime}$. Moreover,

$$
i_{n} \equiv i_{n}^{\prime}\left(\bmod g^{*}\right)
$$

We conclude that

$$
h\left(x_{1}, \ldots, x_{n}\right)=x_{n}^{c} h^{*}\left(x_{1}, \ldots, x_{n-1}, x_{n}^{g^{*}}\right)
$$

and

$$
h^{*}\left(x_{1}, \ldots, x_{n-1}, x_{n}^{g^{*}}\right) \in P
$$

It is easy to see

$$
\begin{aligned}
h^{*}\left(x_{1}, \ldots, x_{n-1}, x_{n}^{g^{*}}\right)^{p^{m}} & =\bar{h}\left(x_{1}, \ldots, x_{n-1},\left(x_{n}^{g^{*}}\right)^{p^{m}}\right) \\
& =h^{\prime}\left(x_{1}, \ldots, x_{n-1}\right) \quad\left(\bmod f_{n-1}\right)
\end{aligned}
$$

and

$$
h^{\prime}\left(x_{1}, \ldots, x_{n-1}\right) \in P \cap k\left[x_{1}, \ldots, x_{n-1}\right]=Q .
$$

Putting the above arguments together we get $h \in \sqrt{\left(f_{1}, \ldots, f_{n-1}\right)}$. Q.E.D.

Corollary. Let $\mathbf{A}_{k}^{n} \subset \mathbf{P}_{k}^{n}$ by dehomogenizing $x_{n+1}=1$. For the monomial curve $C$ this means setting $u=1$. The binomials $\left(f_{1}, \ldots, f_{n-1}\right)$ define the affine piece of $C$ and only pass through the point $(1,0, \ldots, 0)$ at $\infty$. Hence the homogenized polynomials $f_{1}^{\#}, \ldots, f_{n-1}^{\#}$ define the projective curve $C$.

2. Let $C$ be a curve in $\mathbf{P}_{k}^{n}$, where $k$ is an algebraically closed field of characteristic $p$. Let $\tau$ be a linear projection $\mathbf{P}_{k}^{n} \rightarrow \mathbf{P}_{k}^{2}$ with center $D$. Suppose $D \cap C=\varnothing, C$ is 
birational to $\tau(C)$, and $\tau(C)$ has only cusps as singularities. Then we have

THEOREM 2. Under the above assumption, $C$ is a set-theoretic complete intersection.

Proof. Choose $L_{\infty} \subset \mathbf{P}_{k}^{2}$ so that $L_{\infty} \cap C^{\prime}$ consists of $d$ distinct points where $C^{\prime}=\tau(C)$ and $d=$ degree of $C^{\prime}=$ order of $C$. Take $L_{\infty}$ as the line at $\infty$, the complement of $L_{\infty}=\mathbf{A}_{k}^{2}$. Let $f\left(x_{1}, x_{2}\right)=$ the defining equation of $C^{\prime} \cap \mathbf{A}_{k}^{2}$. Then $d=\operatorname{deg} f\left(x_{1}, x_{2}\right)$ and $\partial f\left(x_{1}, x_{2}\right)=$ highest homogeneous form has $d$ distinct roots.

Let $H$ be the hyperplane spanned by $L_{\infty}$ and $D$. The hyperplane $H=\tau^{-1}\left(L_{\infty}\right)$. Consider $H$ as the hyperplane at $\infty$ and $\mathbf{P}_{k}^{n}=\mathbf{A}_{k}^{n} \cup H$. Let the coordinate ring $\mathbf{A}_{k}^{\prime \prime}=k\left[x_{1}, x_{2}, \ldots, x_{n}\right]$.

The condition that $C^{\prime}$ has only cusps as singularities implies that the preimage $\tau^{-1}(P)$ consists of a single point $\{Q\}$ for every $P \in C^{\prime}$.

We shall discuss the corresponding problem for the affine pieces $C \cap \mathbf{A}_{k}^{n} \stackrel{\tau}{\rightarrow} C^{\prime} \cap$ $\mathbf{A}_{k}^{2}$ first. Let $I=$ the ideal of $C \cap \mathbf{A}_{k}^{n}, J=\left(f\left(x_{1}, x_{2}\right)\right)$. Let $E$ be the conductor of $k\left[x_{1}, x_{2}\right] / J \hookrightarrow k\left[x_{1}, x_{2}, \ldots, x_{n}\right] / I$. Let $Q_{1}, \ldots, Q_{m}$ be the points belonging to $E$ on $C \cap \dot{\mathbf{A}}_{k}^{n}$ and $P_{1}, \ldots, P_{m}$ be the corresponding points on $C^{\prime} \cap \mathbf{A}_{k}^{2}$. Then at $Q_{1}, \ldots, Q_{n}$ let $\bar{x}_{i}$ be the canonical image of $x_{i}$ in $k\left[x_{1}, \ldots, x_{n}\right] / I$. We have

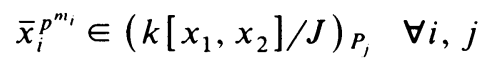

for $m_{i}$ large enough. Hence we get

$$
\bar{x}_{i}^{p^{m_{i}}} \in k\left[x_{1}, x_{2}\right] / J \quad \forall i .
$$

In other words, there are $m_{i}$ and $g_{i}\left(x_{1}, x_{2}\right)$ such that

$$
f_{i}=x_{i}^{p^{m_{i}}}-g_{i}\left(x_{1}, x_{2}\right) \in I \quad \forall i \geqslant 3 \text {. }
$$

We claim that $\left(f, f_{3}, \ldots, f_{n}\right)$ defines $C \cap \mathbf{A}_{k}^{n}$. We follow Cowsik-Nori. Let $g \in I$ and $N \geqslant m_{i} \forall i$. Then clearly

$$
\begin{aligned}
g\left(x_{1}, x_{2}, \ldots, x_{n}\right)^{p^{N}} & =\bar{g}\left(x_{1}, x_{2}, x_{3}^{p^{N}}, \ldots, x_{n}^{p^{N}}\right) \\
& =g^{\prime}\left(x_{1}, x_{2}\right) \quad\left(\bmod f_{3}, \ldots, f_{n}\right)
\end{aligned}
$$

and

$$
g^{\prime}\left(x_{1}, x_{2}\right) \in J=(f)
$$

so that we have $g \in \sqrt{\left(f, f_{3}, \ldots, f_{n}\right)}$.

To finish the proof of the projective case we homogenize $f_{1}, f_{3}, \ldots, f_{n}$ to $f^{\#}$, $f_{3}^{\#}, \ldots, f_{n}^{\#}$. Let the $d$ points at $\infty$ be $\bar{Q}_{1}, \ldots, \bar{Q}_{d}$ and $\bar{P}_{1}, \ldots, \bar{P}_{d}$ for $\mathbf{P}_{k}^{n}$ and $\mathbf{P}_{k}^{2}$, respectively. We claim that $f, f_{3}, \ldots, f_{n}$ can be modified so that $\left(\partial f, \partial f_{3}, \ldots, \partial f_{n}\right)$ only pass through $\bar{Q}_{1}, \ldots, \bar{Q}_{d}$. There are two cases to be considered: Case I. $\operatorname{deg} f_{i}>p^{m_{i}}$. Case II. $\operatorname{deg} f_{i}=p^{m_{i}}$. In Case I, since $f_{i}$ indeed passes through $\bar{Q}_{1}, \ldots, \bar{Q}_{d}$, we conclude at once that $g_{i}\left(x_{1}, x_{2}\right)$ passes through $\bar{P}_{1}, \ldots, \bar{P}_{d}$. In other words

$$
\partial f\left(x_{1}, x_{2}\right) \mid \partial g_{i}\left(x_{1}, x_{2}\right) \text {. }
$$

So we may modify $g_{i}\left(x_{1}, x_{2}\right)$ by $f\left(x_{1}, x_{2}\right)$ to reduce the degree of $f_{i}$. Finally we may assume we are in Case II. Now the polynomial $\partial f_{i}$ is an inseparable equation in $x_{i}$ for $i \geqslant 3$. So there is only one possible solution for $x_{i}$ if the values of $x_{1}, x_{2}$ are fixed. Thus there is only one point lying over $\bar{P}_{1}, \ldots, \bar{P}_{d}$, namely, $\bar{Q}_{1}, \ldots, \bar{Q}_{d}$. Q.E.D. 


\section{REFERENCES}

1. R. Cowsik and M. Nori, Affine curtes in characteristic $p$ are set-theoretic complete intersections, Invent. Math. 45 (1978), 111-114.

2. D. Ferrand, Set theoretic complete intersections in characteristic $p>0$, Lecture Notes in Math., vol. 732. Springer-Verlag, 1979, pp. 82-89.

3. R. Hartshorne, Complete intersections in characteristic $p>0$, Amer. J. Math. 101 (1979), 380-383.

Dipartment of Mathematics, Purdue University, West Lafayette, Indiana 47907 